\title{
General System of Nonconvex Variational Inequalities and Parallel Projection Method
}

\author{
Balwant Singh Thakur* And Suja Varghese
}

\begin{abstract}
Using the prox-regularity notion, we introduce and study a system of general nonconvex variational inequalities. Using the parallel projection technique, we suggest and analyze a three-step iterative method for this system. We establish a convergence result for the proposed iteration method. We obtain some known results as a particular case.
\end{abstract}

\section{Introduction AND PRELIMINARIES}

General variational inequality was introduced and studies by Noor [9]. It was a significant generalization of the variational inequalities, which was introduced and studied by Stampacchia [15] in 1964. The set involved in the above studies are convex. For application point of view, getting convex sets is itself is a difficult problem, most of the times the set involved is not convex. To overcome the difficulty caused by the nonconvexity of the set, Noor [12] considered uniformly prox-regular sets and introduced a new class of variational inequality called the general nonconvex variational inequality. The prox-regular sets are nonconvex and include the convex sets as special cases; (see [4]).

In recent years several numerical method appeared for solving variational inequalities. These includes projection method and their variant forms, which was mainly due to Sibony [14]. Iterative schemes to solve nonconvex variational problems was studied by Bounkhel et al. [3]. The projection method is an important technique for the approximate solvability of variational inequalities. The main limitation of projection method is that the convergence analysis requires the restrictive condition that the operators involved must be strongly monotone. This strict condition rule out many applications of the projection method for a wide class of problems. Gabay [5] and Tseng [16] used cocoercive operators for projection method. It is

2010 Mathematics Subject Classification. 47J20, 65K10, 65K15, 90C33.

Key words and phrases. System of nonconvex general variational inequality, fixed point problem, parallel algorithm, proximal normal cone, relaxed cocoercive mapping.

${ }^{*}$ Corresponding author. 
also found that two-step and three-step iteration method preform better than one-step method, see $[10,11]$. Now a days implementation of iterative algorithms have mainly been implemented through computer systems and we also have modern multiprocessor systems in which different processor can work simultaneously. When we apply two/three step sequential iteration process we use only one processor at a time, so fails to explore the full capacity of multiprocessor. If we define parallel iterative algorithm, we can compute all the steps simultaneously on different processors, and this will take less computational time than the sequential iteration which uses only one processor at a time; (see $[1,2,6,7]$ for details in parallel computation).

Let $H$ be a real Hilbert space whose inner product and norm are denoted by $\langle.,$.$\rangle and \|$.$\| respectively. Let K$ be a nonempty subset of $H$. We denote by $d(., K)$ the usual distance function to the subset $K$, i.e. $d(x, K)=\inf _{y \in K}\|x-y\|$. Now we recall some well-known definitions and results of nonlinear convex analysis and nonsmooth analysis.

Definition 1.1. [4] Let $x \in H$ be a point not lying in $K$. Let $y \in K$ is a point whose distance to $x$ is minimal, i.e. $d(x, K)=\|x-y\|$, then $y$ is called a closest point or a projection of $x$ onto $K$. The set of all such closest points is denoted by $\operatorname{proj}_{K}(x)$; that is,

$$
\operatorname{proj}_{K}(x)=\{y \in K: d(x, K)=\|x-y\|\} .
$$

Also, $y \in \operatorname{proj}_{K}(x)$ if and only if $\{y\} \subset K \cap \bar{B}\{x ;\|x-y\|\}$ and $K \cap$ $B\{x ;\|x-y\|\}=\emptyset$. The vector $x-y$ is called a proximal normal direction to $K$ at $y$. Any nonnegative multiple $z=\alpha(x-y), \alpha \geq 0$ of such a vector is called a proximal normal to $K$ at $x$. The set of all $z$ obtainable in this manner is turned as proximal normal cone to $K$ at $x$ and is denoted by $N_{K}^{p}(x)$.

Definition 1.2 ([13]). The proximal normal cone to $K$ at $x \in H$ is given by

$$
N_{K}^{p}(x)=\left\{z \in H: \exists \alpha>0 ; x \in \operatorname{proj}_{K}(x+\alpha z)\right\} .
$$

The proximal normal cone $N_{K}^{p}(x)$ has the following characterization.

Lemma 1.3. [13, Proposition 1.5] Let $K$ be a nonempty subset of $H$. Then a vector $z \in N_{K}^{p}(x)$ if and only if there exists a constant $\alpha=\alpha(z, x) \geq 0$ such that

$$
\langle z, y-x\rangle \leq \alpha\|y-x\|^{2}, \forall y \in K
$$

Clarke et al.[4] and Poliquin et al.[13] introduced and studied a new class of nonconvex sets called uniformly prox-regular sets.

Definition 1.4. For a given $r \in(0, \infty]$, a subset $K$ of $H$ is said to be uniformly prox-regular with respect to $r$ ( or $r$-uniformly prox-regular) if and only if, for all $x \in K$ and for all $0 \neq z \in N_{K}^{p}(x)$, one has

$$
\left\langle\frac{z}{\|z\|}, y-x\right\rangle \leq \frac{1}{2 r}\|y-x\|^{2} \text { for all } y \in K \text {. }
$$


We use the convention that $\frac{1}{r}=0$ when $r=+\infty$.

A closed subset of a Hilbert space is convex if and only if it is proximally smooth of radius $r>0$. Thus, in view of Definition 1.4, for the case of $r=\infty$, the notion of uniform prox-regularity and convexity of $K$ coincide. It is known that the class of uniformly prox-regular set is sufficiently large to include the class of convex sets, $p$-convex sets, $C^{1,1}$ submanifolds of $H$, the images under a $C^{1,1}$ diffeomorphism of convex sets and many other nonconvex sets.

Now recall the well known proposition which summarizes some important properties of the uniformly prox-regularity.

Lemma 1.5. Let $K$ be nonempty closed subset of $H, r \in(0, \infty]$ and $K_{r}=$ $\{x \in H: d(x, K)<r\}$. If $K$ is uniformly $r$-prox-regular, then the following holds:

(i) For all $x \in K_{r}$, set $\operatorname{proj}_{K}(x) \neq \emptyset$.

(ii) For all $s \in(0, r), \operatorname{proj}_{K}$ is Lipschitz continuous with constant $\frac{r}{r-s}$ on $K_{s}$.

(iii) The proximal normal cone $N_{K}^{p}(x)$ is closed as a set valued mapping.

Let $H$ be a Hilbert space and $K$ a closed convex subset of $H$. Consider the following problem:

Find $x^{*}, y^{*}, z^{*} \in K$ such that $g\left(x^{*}\right), g\left(y^{*}\right), g\left(z^{*}\right) \in K$,

$$
\left\{\begin{array}{l}
\left\langle\rho T_{1}\left(y^{*}\right)+g\left(x^{*}\right)-g\left(y^{*}\right), g(x)-g\left(x^{*}\right)\right\rangle \geq 0, \\
\left\langle\eta T_{2}\left(z^{*}\right)+g\left(y^{*}\right)-g\left(z^{*}\right), g(x)-g\left(y^{*}\right)\right\rangle \geq 0, \\
\left\langle\sigma T_{3}\left(x^{*}\right)+g\left(z^{*}\right)-g\left(x^{*}\right), g(x)-g\left(z^{*}\right)\right\rangle \geq 0,
\end{array}\right.
$$

for all $x \in K, g(x) \in K$, where $g: H \rightarrow H$ be a given mapping, $T_{1}, T_{2}, T_{3}$ : $K \rightarrow H$ are nonlinear operators and $\rho, \eta, \sigma$ are nonnegative real numbers. We call problem (1.1) as system of general variational inequalities (SGVI).

By the definition of the Normal cone, we now reformulate (SGVI) as follows :

$$
\left\{\begin{array}{l}
0 \in \rho T_{1}\left(y^{*}\right)+g\left(x^{*}\right)-g\left(y^{*}\right)+N_{K}\left(g\left(x^{*}\right)\right), \\
0 \in \eta T_{2}\left(z^{*}\right)+g\left(y^{*}\right)-g\left(z^{*}\right)+N_{K}\left(g\left(y^{*}\right)\right), \\
0 \in \sigma T_{3}\left(x^{*}\right)+g\left(z^{*}\right)-g\left(x^{*}\right)+N_{K}\left(g\left(z^{*}\right)\right) .
\end{array}\right.
$$

By replacing the usual normal cone by proximal normal cone, we now introduce the generalized version of problem (1.2) which we call system of nonconvex general variational inequalities (SNGVI).

Let $H$ be a Hilbert space and $K$ a uniformly $r$-prox-regular subset of $H$. We will consider the following problem: 
Find $x^{*}, y^{*}, z^{*} \in K$ such that $g\left(x^{*}\right), g\left(y^{*}\right), g\left(z^{*}\right) \in K$,

$$
\begin{aligned}
& 0 \in \rho T_{1}\left(y^{*}\right)+g\left(x^{*}\right)-g\left(y^{*}\right)+N_{K}^{p}\left(g\left(x^{*}\right)\right), \\
& 0 \in \eta T_{2}\left(z^{*}\right)+g\left(y^{*}\right)-g\left(z^{*}\right)+N_{K}^{p}\left(g\left(y^{*}\right)\right), \\
& 0 \in \sigma T_{3}\left(x^{*}\right)+g\left(z^{*}\right)-g\left(x^{*}\right)+N_{K}^{p}\left(g\left(z^{*}\right)\right) .
\end{aligned}
$$

Lemma 1.6. Let $K$ a uniformly $r$-prox-regular subset of $H$, then $x^{*}, y^{*}, z^{*} \in$ $H$ with $g\left(x^{*}\right), g\left(y^{*}\right), g\left(z^{*}\right) \in K$ is a solution of (SNGVI) if and only if

$$
\begin{aligned}
& g\left(x^{*}\right)=\operatorname{proj}_{K}\left(g\left(y^{*}\right)-\rho T_{1}\left(y^{*}\right)\right), \\
& g\left(y^{*}\right)=\operatorname{proj}_{K}\left(g\left(z^{*}\right)-\eta T_{2}\left(z^{*}\right)\right), \\
& g\left(z^{*}\right)=\operatorname{proj}_{K}\left(g\left(x^{*}\right)-\sigma T_{3}\left(x^{*}\right)\right),
\end{aligned}
$$

provided that

$$
\begin{array}{ll}
0<\rho \leq \frac{s}{1+\left\|T_{1}\left(y^{*}\right)\right\|}, & 0<\eta \leq \frac{s}{1+\left\|T_{2}\left(z^{*}\right)\right\|}, \\
0<\sigma \leq \frac{s}{1+\left\|T_{3}\left(x^{*}\right)\right\|}, & s \in(0, r) .
\end{array}
$$

Proof. Using (1.3), and the fact that $\operatorname{proj}_{K}=\left(I+N_{K}^{p}\right)^{-1}$, we have

$$
\begin{aligned}
0 & \in \rho T_{1}\left(y^{*}\right)+g\left(x^{*}\right)-g\left(y^{*}\right)+N_{K}^{p}\left(g\left(x^{*}\right)\right) \\
& \Leftrightarrow g\left(y^{*}\right)-\rho T_{1}\left(y^{*}\right) \in g\left(x^{*}\right)+N_{K}^{p}\left(g\left(x^{*}\right)\right)=\left(I+N_{K}^{p}\right)\left(g\left(x^{*}\right)\right) \\
& \Leftrightarrow g\left(x^{*}\right)=\operatorname{proj}_{K}\left(g\left(y^{*}\right)-\rho T_{1}\left(y^{*}\right)\right),
\end{aligned}
$$

where $I$ is the identity mapping.

Similarly, using (1.4) and (1.5), we have

$$
\begin{aligned}
& g\left(y^{*}\right)=\operatorname{proj}_{K}\left(g\left(z^{*}\right)-\eta T_{2}\left(z^{*}\right)\right) \\
& g\left(z^{*}\right)=\operatorname{proj}_{K}\left(g\left(x^{*}\right)-\sigma T_{3}\left(x^{*}\right)\right) .
\end{aligned}
$$

This completes the proof.

Lemma 1.6 implies that (SNGVI) is equivalent to the fixed point problem. This alternative equivalent formulation is very useful from the numerical point of view. This fixed point formulation suggest the following iteration method to solve (SNGVI)

$$
\left\{\begin{array}{l}
g\left(x_{k+1}\right)=\operatorname{proj}_{K}\left(g\left(y_{k}\right)-\rho T_{1}\left(y_{k}\right)\right), \\
g\left(y_{k+1}\right)=\operatorname{proj}_{K}\left(g\left(z_{k}\right)-\eta T_{2}\left(z_{k}\right)\right), \\
g\left(z_{k+1}\right)=\operatorname{proj}_{K}\left(g\left(x_{k}\right)-\sigma T_{3}\left(x_{k}\right)\right) .
\end{array}\right.
$$

where $\rho, \eta, \sigma$ are positive reals, satisfying certain conditions.

We now recall some definition, which will be used in the main result. 
Definition 1.7. A mapping $H \rightarrow H$ is called to be $\zeta$-expansive if for all $x, y \in H$, there exists a constant $\zeta>0$, such that

$$
\|g(x)-g(y)\| \geq \zeta\|x-y\| .
$$

Definition 1.8. An operator $T: H \rightarrow H$ with respect to an arbitrary operator $g$ is said to be :

(i) $(g, \nu)$ strongly monotone, if for all $x, y \in H$, there exists a constant $\nu>0$ such that

$$
\langle T(x)-T(y), g(x)-g(y)\rangle \geq \nu\|g(x)-g(y)\|^{2},
$$

(ii) $(g, \omega)$ cocoercive, if for all $x, y \in H$, there exists a constant $\omega>0$ such that

$$
\langle T(x)-T(y), g(x)-g(y)\rangle \geq \omega\|T x-T y\|^{2},
$$

(iii) relaxed $(g, \omega, t)$ cocoercive, if for all $x, y \in H$, there exists constants $t>0$ and $\omega>0$ such that

$$
\langle T(x)-T(y), g(x)-g(y)\rangle \geq-\omega\|T x-T y\|^{2}+t\|g(x)-g(y)\|^{2},
$$

(iv) $(g, \mu)$ Lipschitz continuous, if for all $x, y \in H$, there exists a constant $\mu>0$ such that

$$
\|T(x)-T(y)\| \leq \mu\|g(x)-g(y)\| .
$$

\section{MAIN RESUlT}

We now present, a result for the approximation-solvability of the (SNGVI) problem using parallel algorithm 1.6. In what follows we assume that $K$ is a uniformly $r$-prox-regular subset of $H$ with $r>0$, also let $s \in(0, r)$ and set $\delta=\frac{r}{r-s}$.

Theorem 2.1. Assume that $g: H \rightarrow H$ be $\zeta$-expansive mapping and $T_{i}: K \rightarrow H$ be relaxed $\left(g, \omega_{i}, t_{i}\right)$-cocoercive and $\left(g, \mu_{i}\right)$ Lipschitz continuous mappings satisfying $\delta\left(\omega_{i} \mu_{i}^{2}+t_{i}\right)>\mu_{i} \sqrt{\delta^{2}-1}, 0<\delta \theta_{i}<1$, for $i=1,2,3$. Suppose that $x^{*}, y^{*}, z^{*} \in K$ form a solution to (SNGVI), then the sequence $\left\{x_{k}, y_{k}, z_{k}\right\}$ generated by (1.6) strongly converges to $\left(x^{*}, y^{*}, z^{*}\right)$, provided that the following conditions are satisfied:

$$
\begin{aligned}
& \Theta_{1}-\Delta_{1} \leq \rho \leq \min \left\{\Theta_{1}+\Delta_{1}, \frac{s}{1+\left\|T_{1}\left(y_{n}\right)\right\|}, \frac{s}{1+\left\|T_{1}\left(y^{*}\right)\right\|}\right\}, \\
& \Theta_{2}-\Delta_{2} \leq \eta \leq \min \left\{\Theta_{2}+\Delta_{2}, \frac{s}{1+\left\|T_{2}\left(z_{n}\right)\right\|}, \frac{s}{1+\left\|T_{2}\left(z^{*}\right)\right\|}\right\}, \\
& \Theta_{3}-\Delta_{3} \leq \sigma \leq \min \left\{\Theta_{3}+\Delta_{3}, \frac{s}{1+\left\|T_{3}\left(x_{n}\right)\right\|}, \frac{s}{1+\left\|T_{3}\left(x^{*}\right)\right\|}\right\},
\end{aligned}
$$


$\Theta_{i}=\frac{\omega_{i} \mu_{i}^{2}+t_{i}}{\mu_{i}^{2}}, \Delta_{i}=\frac{\sqrt{\delta^{2}\left(\omega_{i} \mu_{i}^{2}+t_{i}\right)^{2}-\mu_{i}^{2}\left(\delta^{2}-1\right)}}{\delta \mu_{i}^{2}}$, for $i=1,2,3$.

$\theta_{1}=\sqrt{1+2 \rho \omega_{1} \mu_{1}^{2}-2 \rho t_{1}+\rho^{2} \mu_{1}^{2}}, \theta_{2}=\sqrt{1+2 \eta \gamma_{2} \mu_{2}^{2}-2 \eta t_{2}+\eta^{2} \mu_{2}^{2}}$ and $\theta_{3}=\sqrt{1+2 \sigma \gamma_{3} \mu_{3}^{2}-2 \sigma t_{3}+\sigma^{2} \mu_{3}^{2}}$.

Proof. Since $\left(x^{*}, y^{*}, z^{*}\right)$ is a solution of (SNGVI), from the conditions on the parameters $\rho, \eta$ and $\sigma$, we have

$$
\begin{aligned}
& g\left(x^{*}\right)=\operatorname{proj}_{K}\left(g\left(y^{*}\right)-\rho T_{1}\left(y^{*}\right)\right), \\
& g\left(y^{*}\right)=\operatorname{proj}_{K}\left(g\left(z^{*}\right)-\eta T_{2}\left(z^{*}\right)\right), \text { and } \\
& g\left(z^{*}\right)=\operatorname{proj}_{K}\left(g\left(x^{*}\right)-\sigma T_{3}\left(x^{*}\right)\right) .
\end{aligned}
$$

Using (1.6), we can write

$$
\begin{aligned}
\left\|g\left(x_{k+1}\right)-g\left(x^{*}\right)\right\| & =\left\|\operatorname{proj}_{K}\left(g\left(y_{k}\right)-\rho T_{1}\left(y_{k}\right)\right)-\operatorname{proj}_{K}\left(g\left(y^{*}\right)-\rho T_{1}\left(y^{*}\right)\right)\right\| \\
& \leq \delta\left\|\left(g\left(y_{k}\right)-\rho T_{1}\left(y_{k}\right)\right)-\left(g\left(y^{*}\right)-\rho T_{1}\left(y^{*}\right)\right)\right\| .
\end{aligned}
$$

Because of choice of $\rho$ we have $g\left(y_{k}\right)-\rho T_{1}\left(y_{k}\right)$ and $g\left(y^{*}\right)-\rho T_{1}\left(y^{*}\right)$ belongs to $K_{s}$. Since $T_{1}$ is relaxed $\left(g, \omega_{1}, t_{1}\right)$ cocoercive and $\left(g, \mu_{1}\right)$ Lipschitzian, we have

$$
\begin{aligned}
&\left\|g\left(y_{k}\right)-g\left(y^{*}\right)-\rho\left(T_{1}\left(y_{k}\right)-T_{1}\left(y^{*}\right)\right)\right\|^{2} \\
&=\left\|g\left(y_{k}\right)-g\left(y^{*}\right)\right\|^{2}-2 \rho\left\langle T_{1}\left(y_{k}\right)-T_{1}\left(y^{*}\right), g\left(y_{k}\right)-g\left(y^{*}\right)\right\rangle \\
&+\rho^{2}\left\|T_{1}\left(y_{k}\right)-T_{1}\left(y^{*}\right)\right\|^{2} \\
& \leq\left\|g\left(y_{k}\right)-g\left(y^{*}\right)\right\|^{2}+2 \rho \omega_{1}\left\|T_{1}\left(y_{k}\right)-T_{1}\left(y^{*}\right)\right\|^{2} \\
&-2 \rho t_{1}\left\|g\left(y_{k}\right)-g\left(y^{*}\right)\right\|^{2}+\rho^{2}\left\|T_{1}\left(y_{k}\right)-T_{1}\left(y^{*}\right)\right\|^{2} \\
& \leq\left\|g\left(y_{k}\right)-g\left(y^{*}\right)\right\|^{2}+2 \rho \omega_{1} \mu_{1}^{2}\left\|g\left(y_{k}\right)-g\left(y^{*}\right)\right\|^{2} \\
&-2 \rho t_{1}\left\|g\left(y_{k}\right)-g\left(y^{*}\right)\right\|^{2}+\rho^{2} \mu_{1}^{2}\left\|g\left(y_{k}\right)-g\left(y^{*}\right)\right\|^{2} \\
&= {\left[1+2 \rho \omega_{1} \mu_{1}^{2}-2 \rho t_{1}+\rho^{2} \mu_{1}^{2}\right]\left\|g\left(y_{k}\right)-g\left(y^{*}\right)\right\|^{2} . }
\end{aligned}
$$

By (2.1) and (2.2), we have

$$
\left\|g\left(x_{k+1}\right)-g\left(x^{*}\right)\right\| \leq \delta \theta_{1}\left\|g\left(y_{k}\right)-g\left(y^{*}\right)\right\|,
$$

where $\theta_{1}=\sqrt{1+2 \rho \omega_{1} \mu_{1}^{2}-2 \rho t_{1}+\rho^{2} \mu_{1}^{2}}$.

Similarly, since $T_{2}$ is relaxed $\left(g, \gamma_{2}, t_{2}\right)$ cocoercive and $\left(g, \mu_{2}\right)$ Lipschitzian, we have

$$
\left\|g\left(y_{k+1}\right)-g\left(y^{*}\right)\right\| \leq \delta \theta_{2}\left\|g\left(z_{k}\right)-g\left(z^{*}\right)\right\|,
$$

where $\theta_{2}=\sqrt{1+2 \eta \gamma_{2} \mu_{2}^{2}-2 \eta t_{2}+\eta^{2} \mu_{2}^{2}}$.

Also, since $T_{3}$ is relaxed $\left(g, \gamma_{3}, t_{3}\right)$ cocoercive and $\left(g, \mu_{3}\right)$ Lipschitzian, we have

$$
\left\|g\left(z_{k+1}\right)-g\left(z^{*}\right)\right\| \leq \delta \theta_{3}\left\|g\left(x_{k}\right)-g\left(x^{*}\right)\right\|,
$$

where $\theta_{3}=\sqrt{1+2 \sigma \gamma_{3} \mu_{3}^{2}-2 \sigma t_{3}+\sigma^{2} \mu_{3}^{2}}$. 
From (2.3), (2.4) and (2.5), it follows that

$$
\begin{aligned}
& \left\|g\left(x_{k+1}\right)-g\left(x^{*}\right)\right\|+\left\|g\left(y_{k+1}\right)-g\left(y^{*}\right)\right\|+\left\|g\left(z_{k+1}\right)-g\left(z^{*}\right)\right\| \\
\leq & \delta \theta_{3}\left\|g\left(x_{k}\right)-g\left(x^{*}\right)\right\|+\delta \theta_{1}\left\|g\left(y_{k}\right)-g\left(y^{*}\right)\right\|+\delta \theta_{2}\left\|g\left(z_{k}\right)-g\left(z^{*}\right)\right\| \\
\leq & \max \left\{\delta \theta_{1}, \delta \theta_{2}, \delta \theta_{3}\right\}\left(\left\|g\left(x_{k}\right)-g\left(x^{*}\right)\right\|+\right. \\
& \left.+\left\|g\left(y_{k}\right)-g\left(y^{*}\right)\right\|+\left\|g\left(z_{k}\right)-g\left(z^{*}\right)\right\|\right) \\
(2.6)=\lambda\left(\left\|g\left(x_{k}\right)-g\left(x^{*}\right)\right\|+\right. & \left.\left\|g\left(y_{k}\right)-g\left(y^{*}\right)\right\|+\left\|g\left(z_{k}\right)-g\left(z^{*}\right)\right\|\right),
\end{aligned}
$$

where $\lambda=\max \left\{\delta \theta_{1}, \delta \theta_{2}, \delta \theta_{3}\right\}<1$.

Now, define the norm $\|\cdot\|_{1}$ on $H \times H \times H$ by

$$
\|(x, y, z)\|_{1}=\|x\|+\|y\|+\|z\| \text {, for all }(x, y, z) \in H \times H \times H .
$$

Then $\left(H \times H \times H,\|\cdot\|_{1}\right)$ is a Banach space. Hence, (2.6) implies that

$$
\begin{aligned}
& \left\|\left(g\left(x_{k+1}\right), g\left(y_{k+1}\right), g\left(z_{k+1}\right)\right)-\left(g\left(x^{*}\right), g\left(y^{*}\right), g\left(z^{*}\right)\right)\right\|_{1} \\
& \quad \leq \lambda\left\|\left(g\left(x_{k}\right), g\left(y_{k}\right), g\left(z_{k}\right)\right)-\left(g\left(x^{*}\right), g\left(y^{*}\right), g\left(z^{*}\right)\right)\right\|_{1} \\
& \leq \lambda^{k}\left\|\left(g\left(x_{1}\right), g\left(y_{1}\right), g\left(z_{1}\right)\right)-\left(g\left(x^{*}\right), g\left(y^{*}\right), g\left(z^{*}\right)\right)\right\|_{1} .
\end{aligned}
$$

Since $\lambda<1$, we get from (2.7) that

$$
\lim _{k \rightarrow \infty}\left\|\left(g\left(x_{k}\right), g\left(y_{k}\right), g\left(z_{k}\right)\right)-\left(g\left(x^{*}\right), g\left(y^{*}\right), g\left(z^{*}\right)\right)\right\|_{1}=0 .
$$

Therefore, we obtain

$$
\begin{aligned}
& \lim _{k \rightarrow \infty}\left\|g\left(x_{k}\right)-g\left(x^{*}\right)\right\|=0, \\
& \lim _{k \rightarrow \infty}\left\|g\left(y_{k}\right)-g\left(y^{*}\right)\right\|=0, \\
& \lim _{k \rightarrow \infty}\left\|g\left(z_{k}\right)-g\left(z^{*}\right)\right\|=0 .
\end{aligned}
$$

Hence, sequences $\left\{g\left(x_{k}\right)\right\},\left\{g\left(y_{k}\right)\right\},\left\{g\left(z_{k}\right)\right\}$ converges to $g\left(x^{*}\right), g\left(y^{*}\right)$ and $g\left(z^{*}\right)$ respectively. Since $g$ is $\zeta$-expansive, sequences $\left\{x_{k}\right\},\left\{y_{k}\right\}$ and $\left\{z_{k}\right\}$ converges to $x^{*}, y^{*}$ and $z^{*}$ respectively.

This completes the proof.

For $z^{*}=x^{*}$ and $\eta=\sigma$ and $T_{2}=T_{3}$, the (SNGVI) reduces to the following system of nonconvex variational inequality problem:

Find $x^{*}, y^{*}, z^{*} \in K$ such that $g\left(x^{*}\right), g\left(y^{*}\right), g\left(z^{*}\right) \in K$,

$$
\begin{aligned}
& 0 \in \rho T_{1}\left(y^{*}\right)+g\left(x^{*}\right)-g\left(y^{*}\right)+N_{K}^{p}\left(g\left(x^{*}\right)\right), \\
& 0 \in \eta T_{2}\left(x^{*}\right)+g\left(y^{*}\right)-g\left(x^{*}\right)+N_{K}^{p}\left(g\left(y^{*}\right)\right) .
\end{aligned}
$$

System (2.8) appears to be new one.

If we take $T_{1}=T_{2}$ and $g=$ identity mapping in the system (2.8), then we have following system of nonconvex variational inequality:

Find $x^{*}, y^{*} \in K$ such that

$$
\begin{aligned}
& 0 \in \rho T\left(y^{*}\right)+x^{*}-y^{*}+N_{K}^{p}\left(x^{*}\right), \\
& 0 \in \eta T\left(x^{*}\right)+y^{*}-x^{*}+N_{K}^{p}\left(y^{*}\right) .
\end{aligned}
$$


Remark 1. Moudafi [8] studied the system (2.9) and used following extended iteration :

$$
\begin{aligned}
x_{k+1} & =\left(1-\alpha_{k}\right) x_{k}+\alpha_{k} \operatorname{proj}_{K}\left(y_{k}-\rho T\left(y_{k}\right)\right), \\
y_{k} & =\left(1-\beta_{k}\right) x_{k}+\beta_{k} \operatorname{proj}_{K}\left(x_{k}-\eta T\left(x_{k}\right)\right),
\end{aligned}
$$

where $\rho, \eta$ are positive reals, $0 \leq \alpha_{k}, \beta_{k} \leq 1$ and $\sum_{k=0}^{\infty} \alpha_{k} \beta_{k}=\infty$.

Since $K$ is not convex, the iteration is not well defined, unless $\alpha_{k}, \beta_{k}=1$.

Theorem 2.1 improves and generalizes Theorem 2.1 of [8] for the problem (SNGVI) and parallel algorithm considered here.

Remark 2. Consider the particular case where $r=+\infty$, we have $\delta=1$ and we can recover Theorem 3.1 of Verma [18] and Theorem 2.1 of Verma [17] from Theorem 2.1.

\section{REFERENCES}

[1] G. Baudet, Asynchronous iterative methods for multiprocessors, J. Assoc. Comput. Mach. 25 (1978), 226-244.

[2] D. Bertsekas, J. Tsitsiklis, Parallel and Distributed Computation, Numerical Methods, Prentice-Hall, Englewood Cliffs, NJ, 1989.

[3] M. Bounkhel, L. Tadj, A. Hamdi, Iterative schemes to solve nonconvex variational problems, J. Inequal. Pure Appl. Math. 4(1) (2003), Art. 14.

[4] F.H. Clarke, Y.S. Ledyaev, P.R. Wolenski, Nonsmooth Analysis and Control Theory, Springer-Verlag, Berlin, 1998.

[5] D.Gabay, Applications of the method of multipliers to variational inequalities, In Augmented Lagrangian Methods : Applications to the Solution of Boundary Value Problems, (Eds. M.Fortin and R.Glowinski),pp.299-331, North Hollan, Amsterdam, (1983).

[6] K.-H. Hoffmann, J. Zou, Parallel algorithms of Schwarz variant for variational inequalities, Number. Funct. Anal. Optim. 13 (1992), 449-462.

[7] K.-H. Hoffmann, J. Zou, Parallel solution of variational inequality problems with nonlinear source terms. IMA J. Numer. Anal. 16 (1996), 31-45.

[8] A. Moudafi, Projection methods for a system of nonconvex variational inequalities, Nonlinear Anal. 71 (2009), 517-520.

[9] M.A. Noor, General variational inequaltities, Appl. Math. Lett. 1 (1988), 119-121.

[10] M.A. Noor, New extragradienmt-type method for general variational inequalities, J. Math. Anal. Appl. 277 (2003), 379-395.

[11] M.A. Noor, Some developments in general variational inequalities, Appl. Math.. Comput. 152 (2004), 199-277.

[12] M.A. Noor, Iterative methods for general nonconvex variational inequalities, Albanian J. Math. 3 (2009), 117-127.

[13] R.A. Poliquin, R.T. Rockafellar, L. Thibault, Local differentiability of distance functions, Trans. Amer. Math. Soc. 352 (2000), 5231-5249.

[14] M. Sibony, Méthods itératives pour les équations et inéquations aux dérivées partielles non linéaires de type monotone, Calcolo 7 (1970), 65-183. 
[15] G. Stampacchia, Formes bilineaires coercitives sur les ensembles convexes, C. R. Math. Acad. Sci. Paris 258 (1964), 4413-4416.

[16] P.Tseng, On linear convergence of iterative methods for the variational inequality problem, J. Comput. Appl. Math. 60 (1995), 237-252.

[17] R.U. Verma, Projection methods, algorithms and new systems of variational inequalities, Comp. Math. Appl. 41 (2001), 1025-1031.

[18] R.U. Verma, General convergence analysis for two-step projection methods and applications to variational problems, Appl. Math. Lett. 18 (2005), 1286-1292.

\section{Balwant Singh Thakur}

School of Studies in Mathematics

Pt.Ravishankar Shukla University

RAIPUR, 492010

INDIA

E-mail address: balwantst@gmail.com

\section{Suja Varghese}

School of Studies in Mathematics

Pt.Ravishankar Shukla University

RAIPUR, 492010

INDIA

E-mail address: sujavarghesedaniel@gmail.com 